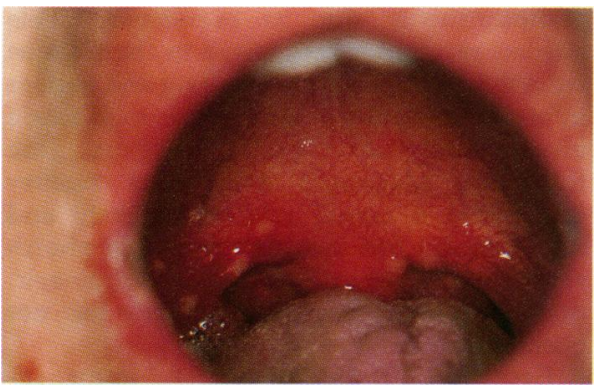

Photograph illustrating multiple herpetic ulcers on oropharynx.

urethral discharge was noted. Microscopy of a Gram stained urethral specimen revealed Gram negative, intracellular diplococci for which he was treated immediately with a dose of $400 \mathrm{mg}$ of ofloxacin. A provisional diagnosis of first episode of herpes simplex virus infection of the oropharyngeal cavity was made, and he was advised to take aciclovir $200 \mathrm{mg} \times 5$ for 5 days.

The patient reported to have always practised homosexual orogenital sex and had never practised anal sex. The sexual history of the recent casual partner was not known. $\mathrm{He}$ admitted to having had a similar sexual contact with another male partner 10 weeks earlier. This was his previous regular partner for 5 years who had no known history of genital herpes.

He returned after 10 days when he was reported to be well and asymptomatic. On examination the oropharyngeal ulcers were noted to be almost healed. A test of cure for $N$ gonorrhoeae from a urethral specimen was reported negative on microscopy. A blood sample was sent for a repeat estimation of HSV antibody.

Herpes simplex virus type 1 (HSV-1) was isolated from the specimen from oropharyngeal ulcers in cell culture. The serum HSV-1 antibody level showed a significant rise from less than 1 in 10 during the first visit to more than 1 in 40 on the tenth day during the follow up visit. This rise in $\mathrm{HSV}-1$ antibody level was consistent with seroconversion for HSV-1.

Microscopy result of $N$ gonorrhoeae from the urethra on his first visit was confirmed on culture. A pharyngeal specimen did not grow $N$ gonorrhoeae.

Isolation of a high proportion of HSV-1 among women with first episode of genital HSV infection was first reported from Sheffield. ${ }^{3}$ Since then an annually increasing prevalence of HSV-1 in female anogenital herpes has been reported by others. ${ }^{4}$ The practice of cunnilingus has been proposed as one of the possible causes of such a trend. ${ }^{5}$ It seems reasonable to assume that such sexual activity could similarly lead to a transmission of HSV from the genital area to the oropharyngeal cavity. In the present case, the occurrence of herpetic lesions in the oropharyngeal cavity within 1 week of unprotected orogenital contact suggests possible transmission of $\mathrm{HSV}-1$ from the genital area to the oropharynx. A first episode of genital HSV-1 infection almost always indicates a true primary infection with HSV. ${ }^{6}$ Thus, seroconversion for HSV-1 in the present case suggests primary infection with this virus and also substantiates the possibility of transmission of $\mathrm{HSV}$ from recent orogenital contact.

The incidence of sexually acquired oropharyngeal herpes due to HSV may increase as a result of increased prevalence of orogenital sexual activity. Because of the risk of transmission of $\mathrm{HSV}$ from asymptomatic viral shedding, the prevalence of HSV carriage and shedding from the oropharynx of sexually active adults needs to be investigated. During counselling, the possibility of acquisition of $\mathrm{HSV}$ infection of the oropharyngeal cavity from the anogenital region, and vice versa, should be discussed.

RANJANA RANI JARVIS

B P GOORNEY

Hope Hospital and

Bolton Centre for Sexual Health

Correspondence to: $\operatorname{Dr} \mathbf{R}$ Rani Jarvis, Bolton Centre for Sexual Health, Royal Bolton Hospital.

1 Russell JM, Azadian BS, Roberts AP, Talboys CA. Pharyngeal flora in a sexually active pop ulation. Int $\mathcal{F} S T D$ AIDS 1995;6:211-5.

2 Tayal SC, Rashid S, Muttu RMS. An audit of pharyngeal infection in patients attending GUM clinic in Sunderland. Int $\mathcal{F}$ STD AIDS 1995;6:60-1.

3 Barton IG, Kinghorn GR, Najem S, AL-Omar $\mathrm{LS}$, Polter CW. Incidence of herpes simplex virus type 1 and 2 in patients with herpes genitalis in Sheffield. Br $\mathcal{F}$ Vener Dis 1982;58: 44-7.

4 Scouler A, Leask BSG, Carrington D. Changing trends in genital herpes due to herpes simplex virus type 1 in Glasgow, 1985-88. Genitourin Med 1990;66:226.

5 Tayal SC, Pattman RS. High prevalence of herpes simplex type 1 in female anogenital herpes simplex in Newcastle-upon Tyne, herpes simplex in Newcastle-upon Tyne,

6 Nahmias AJ, Lee FK, Beckman-Nahmias S Sero-epidemiological sociological patterns of herpes simplex virus infection in the world. Scand $\mathcal{F}$ Infect Dis, (Suppl) 1990;69:19-36.

Accepted for publication 13 May 1997

Syndromic management of sexually transmitted diseases

The WHO has recently recommended the syndromic approach for management of various sexually transmitted diseases. ${ }^{12}$ This is being adapted in an increasing number of countries as it is easy, adaptable, safe, and efficacious. However it has also received some criticisms.

In the Sultanate of Oman, STD prevalence is estimated to be around 121/100 000 and the predominant STDs are gonococcal urethritis, non-specific urethritis, and syphilis (particularly latent syphilis). Infections like granuloma inguinale and lymphogranuloma venereum are uncommon.

In Oman, the syndromic approach for management has recently been adapted as part of the national STD control programme. A national STD manual has also been released and has been made available to all healthcare providers. However, we have made certain modifications to adapt it to the prevailing local conditions, after consideration of the various comments made abou the approach..$^{2-4}$ We would like to highlight these modifications to show how the approach can be successfully adapted to local conditions.

One well meaning criticism is that the WHO recommendation does not include mandatory testing for VDRL and HIV infection. ${ }^{2}$ This is particularly relevant in view of the well established link between HIV infection and other STDs. Since facilities for the transport of blood samples do exist in Oman, we have made it a mandatory requirement for all cases of STD to be investigated with VDRL and ELISA for HIV infection. This will help in detecting latent syphilis/HIV infection.

Another valid criticism is that, with the syndromic approach, data collection and statistical analysis of individual STDs, becomes impossible and would affect future planning. ${ }^{3}$ To overcome this, we have introduced a monthly STD form (in addition to tally sheets for syndromic approach), to be completed by institutions wherein venereologist services are available. The form lists all the sexually transmitted diseases, and would help in collection of data on individual diseases.

A third criticism is that syndromic management results in over treatment and several non-STDs will be treated as STD. ${ }^{2}$ This is? perhaps unavoidable in the syndromic approach. We have sought to counter this by giving a list of non-STDs as a differential diagnosis in our STD manual and by sensitising healthcare providers to the pitfalls in diagnosis, through a series of workshops held $\Omega$ throughout the country.

A well supported argument has been made against the flowchart for vaginal dis-. charge, that without proper vaginal examina- $\overrightarrow{\vec{\omega}}$ tion, lesions located intravaginally will be $\omega$ missed. ${ }^{2}$ In our prevailing social conditions, $c$ vaginal examination by a male doctor is not possible and hence the flow chart is highly $\omega$ relevant. We have further emphasised in our manual that any patient not responding to treatment, or with complications should be referred to the nearest hospital where facili-o ties are available.

Finally, our data analysis showed that latent syphilis was the most common form of syphilis prevalent in the country. Also, a well $\frac{C}{c}$ established system exists in Oman for antenatal screening and screening of blood $\bar{c}$ donors. In addition, all expatriate workers in the country are screened yearly at the time of visa renewal for VDRL and hence management of reported VDRL titres is extremely important. We have included a special sec-o tion on interpretation of VDRL in our STD manual, for this purpose.

As can be seen, our syndromic approach provides a simple model which can be adopted quite easily to the regional situation. We hope our letter will stimulate similaro modifications elsewhere.

MYSORE VENKATARAM A RAOUF AL-SUWAID Department of Dermatology and Genito Urinary Medicine Al Nahdha Hospital and Baushar Polyclinic, Muscat, Oman

Correspondence to: Dr Mysore Nagaraj 118, Muscat, Sultanate of Oman.

1 World Health Organisation. Management of patients with sexually transmitted diseases. $N$ Technical report series No 810-1991. Geneva: WHO.

2 Adler MW. Sexually transmitted disease control in developing countries. Genitourin Med 1996;72:83-8.

3 Bhushan Kumar S. Syndromic management of genital ulcer diseases. (Letter) Genitourin Med 1995;71:193.

4 Van Derm CJ. Syndromic management of genital ulcer disease (reply). Genitourin Med 1996;72:75-6.

Accepted for publication 13 May 1997

\section{Decline in the incidence of HIV test requests in general practices in Amsterdam after 1992}

I wish to report an interesting trend in HIV test seeking behaviour in Amsterdam, where half of all AIDS cases in the Netherlands have occurred. ${ }^{1}$ To assess trends in HIV test seeking behaviour and HIV prevalence in the 
general population in this high risk area, HIV test requests in the general practice sentine network in Amsterdam have been recorded from 1989 to 1992, and from September 1994 to September 1996. The coverage of the network was reduced from $10 \%$ (198992) to $7 \%(1994-5)$ and $2 \%(1995-6)$ of the Amsterdam population, but it remained representative in terms of distribution of practices over the city and sex-age distribution. Through the years, homosexual men accounted for $15-20 \%$ of the HIV test requests and drug users for $3-6 \%$.

The average yearly incidence of test requests between 1989 and 1992 was $5 \cdot 3$ $(4 \cdot 7-5 \cdot 5)$ per 1000 patients, after which it decreased to 3.9 (1994-5) and 2.6 (1995-6). The average percentage of positive test results between 1989 and 1996 was $7 \cdot 0$ $(5 \cdot 9-9 \cdot 0)$, with peaks in $1991(8.6 \%)$ and 1994-5 (9.0\%)

In the nationwide general practice sentine network, which covers about $1 \%$ of the Dutch population, the yearly incidence of HIV test requests per 1000 patients rose steadily from 0.8 (1988) to 1.8 (1993). Of the tests performed, an average of $1 \%$ were positive. $^{2}$

The higher incidence of test requests and positive test results in general practices in Amsterdam confirm the status of Amsterdam as a high risk area for HIV. The marked decline since 1992, in the incidence of test requests in general practices in Amsterdam is interesting, as it contradicts the trend seen elsewhere in the Netherlands. This may well reflect a certain saturation towards HIV testing among the general population in a high risk area.

Towards the end of 1996, the new effective combination treatment for HIV became available. ${ }^{3}$ This is expected to stimulate HIV test requests from individuals who have been at risk for HIV but have not tested before. Given the trend described here, it remains to be seen if a rise in HIV test requests will occur in Amsterdam.

L WIGERSMA Department of General Practice, Academic Medical Centre, Meibergdreef 15 1105 AZ Amsterdam, Netherlands

1 Bindels PJE. Surveillance and survival studies on HIV/AIDS in Amsterdam. PhD thesis. Amsterdam: University of Amsterdam, 1996.

2 Moons MAW, Peters L, Bartelds AIM Kerssens JJ. Concern about AIDS in genera practice. $B M \mathcal{F}$ 1996;312:285-6.

3 Hammer SM. Advances in antiretroviral therapy and viral load monitoring. AIDS 1996;10 (suppl 3):S1-S11.

Accepted for publication 20 May 1997

\section{Genital ulceration revealing a primary} cutaneous anaplastic lymphoma

A 35 year old man first noticed an indurated plaque of the glans penis in 1990. In March 1991, the plaque extended and became painful. Although various antiseptics and antibiotics were applied, he developed an ulceration on the same area in January 1992, which progressively extended to the prepuce. A posthectomy was carried out in March 1992. In June 1992, he had an ulceration of $4 \mathrm{~cm}$ diameter, covered by a plaque of gangrene (fig). He was then admitted in the department of dermatology in Strasbourg. During this 2 year period, TPHA and VDRL were negative four times, as well as ELISA for HIV antibodies. Cultures from the

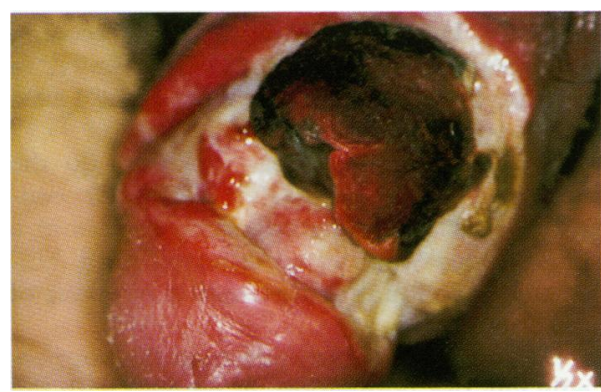

be included in spectrum of the anaplastic large cell lymphoma (CD30 negative). The involvement of large vessels by atypical lymphocytes could be consistent with an angiotrophic lymphoma.

B CRIBIER D LIPSKER E GROSSHANS Clinique Dermatologique, Hôpitaux Universitaires, Strasbourg, France C DUHEM

$C$ CAPESIUS M DICATO

Large ulceration of the penis centred by a plaque of gangrene.

ulceration showed only the presence of Staphylococcus epidermidis. A first biopsy showed in January 1992 a dense granulomatous infiltrate containing numerous plasma cells but no atypical lymphocytes. The prepuce was also examined and did not show malignant changes. In June 1992, the patient suddenly developed an inguinal adenopathy and his general condition worsened, with fever $\left(39^{\circ} \mathrm{C}\right)$ and intense local pain. The histopathological examination of the fat tissue around the enlarged but cytologically normal lymph node showed areas of necrosis, containing a dense infiltrate of malignant cells with highly atypical nuclei and numerous mitoses. The muscular wall of a large vein was infiltrated by neoplastic cells. In the very depth of the genital ulceration, the same malignant cells were also present, which expressed $T$ lymphocyte markers (common leucocyte antigen and CD3) but no B lymphocyte markers (CD20) and CD30 antigen in less than $10 \%$ of cells. The final diagnosis was a primary cutaneous anaplastic large cell lymphoma, which was CD30 negative and showed a marked angiotropism. Total body computed tomography scan and bone marrow biopsy excluded visceral localisation.

The patient was first treated by VP16, cyclophosphamide, vincristine, prednisone, bleomycin, and adriamycin. Nevertheless, there was no regression of the penile ulceration. He was then treated by surgical excision of the persisting lymph nodes and genital necrotic tissue and by a second chemotherapy regimen (dexamethasone, cytarabine, and cisplatin) that allowed a regression of the penile ulceration and a softening of the surgical scar of the groin. Four months later, a local progression of the disease in the inguinal area was noted and he developed a strong increase in ALT and AST levels, circulating blasts and disseminated intravascular coagulation. The patient died in March 1993 because of haemorrhagic complications.

To our knowledge, the occurrence of a primary lymphoma of the penis was never described among malignant tumours causing genital ulcerations. ${ }^{1}$ The diagnosis of lymphoma was extremely difficult in this case because the clinical presentation was unusual and the two first histological examinations failed to show malignant changes. The diagnosis of neoplasia was possible only after locoregional spreading has occurred. This lymphoma probably induced a persistent ulceration because of its angiotropism, as it is described in the "lethal midline granuloma" which is considered to be a T cell lymphoma. ${ }^{2}$ Cutaneous lymphomas other than mycosis fungoides are rare and constitute a heterogeneous group of neoplasms. ${ }^{3}$ Such lymphomas have not yet been described in the male genitalia, but primary lymphomas of the vulva have been recorded. ${ }^{4-6}$ The classification of this disease is difficult. It could

Correspondence to: $\mathrm{B}$ Cribier, $\mathrm{MD}, \mathrm{PhD}$, Clinique Dermatologique des Hôpitaux Universitaires, 1 place de l'Hôpital 67091 Strasbourg Cedex, France.

Mroczkowski TF, Martin DH. Genital ulcer disease. Dermatol Clin 1994;12:753-64.

2 Ishii $\mathrm{Y}$, Yamanaka N, Ogawa K, Yoshida $\mathrm{Y}$, Takami T, Matsuura A. Nasal T cell lymphoma as a type of so-called "lethal midline granuloma". Cancer 1982;50:2336-54.

3 Joly $P$, Thomine E, Lauret P. Cutaneous lymphomas other than mycosis fungoides. Sem Dermatol 1994;13:172-9.

4 Ferrando-Marco J, Martorell MA, Carrato A, Navarro JT. Primary vulvar lymphoma presenting as a clitoridal tumor. Acta Obstet Gynecol Scand 1992;71:543-6.

5 Tuder RM. Vulvar destruction by malignant lymphoma. Gynecol Oncol 1992;45:52-7.

6 Höffkes HG, Schumann A, Uppenkamp $M$, Teschendorf C, Schindler AE, Parwaresch R, et al. Primary non-Hodgkin's lymphom the vagina. Ann Hematol 1995;70:273-6.

Accepted for publication 29 May 1997

High level ciprofloxacin resistant gonorrhoea imported from Russia

Sherrard and colleagues recently expressed concern regarding importation of syphilis and other sexually transmitted diseases among British travellers to Russia and Poland. ${ }^{1}$ We report a case of multiply resistant gonococcal infection imported into the United Kingdom from Russia which failed single dose ciprofloxacin therapy.

A 35 year old British truck driver returned from Russia with a 3 week history of urethral discharge and dysuria following unprotected vaginal intercourse with a casual female partner in Russia. He had not had intercourse with any other partner for 5 months. He received six tablets of unknown content for the discharge in Russia but these had had no effect on his symptoms. His mucopurulent discharge was confirmed on examination and microscopy demonstrated intracellular Gram negative diplococci. He was treated with single dose ciprofloxacin $500 \mathrm{mg}$ and a week's course of doxycycline. He returned after 4 days with no improvement in his symptoms and a repeat Gram stain was still positive for presumptive gonococcal infection. To cover the possibility of ciprofloxacin resistant gonorrhoea, he was given spectinomycin $2 \mathrm{~g}$ intramuscularly. $\mathrm{He}$ was microbiologically and clinically cured of gonorrhoea 1 month later following return from a further episode of travelling.

Neisseria gonorrhoeae was cultured from urethral specimens taken before and after ciprofloxacin therapy. Susceptibility testing with a $30 \mu \mathrm{g}$ nalidixic acid disc predicted ciprofloxacin resistance which was confirmed by the PHLS Gonococcal Reference Laboratory in Bristol where the isolate's ciprofloxacin minimum inhibitory concentration (MIC) was shown to be $16 \mathrm{mg} / \mathrm{l} \mathrm{con-}$ 